\title{
The Contribution of Cosmic Rays Interacting With Molecular Clouds to the Galactic Center Gamma-Ray Excess
}

\author{
Oscar Macias and Chris Gordon \\ Department of Physics and Astronomy, Rutherford Building, \\ University of Canterbury, Private Bag 4800, Christchurch 8140, New Zealand
}

\begin{abstract}
The Fermi-LAT data appear to have an excess of gamma rays from the inner 150 pc of the Galactic Center. The main explanations proposed for this are: an unresolved population of millisecond pulsars (MSPs), dark matter (DM) annihilation, and nonthermal bremsstrahlung produced by a population of electrons interacting with neutral gas in molecular clouds. The first two options have spatial templates well fitted by the square of a generalized Navarro-Frenk-White (NFW) profile with inner slope $\gamma=1.2$. We model the third option with a $20-\mathrm{cm}$ continuum emission Galactic Ridge template. A template based on the HESS residuals is shown to give similar results. The gamma-ray excess is found to be best fit by a combination of the generalized NFW squared template and a Galactic Ridge template. We also find the spectra of each template is not significantly affected in the combined fit and is consistent with previous single template fits. That is the generalized NFW squared spectrum can be fit by either of order 1000 unresolved MSPs or DM with mass around $30 \mathrm{GeV}$, a thermal cross section, and mainly annihilating to $b \bar{b}$ quarks. While the Galactic Ridge continues to have a spectrum consistent with a population of nonthermal electrons whose spectrum also provides a good fit to synchrotron emission measurements. We also show that the current DM fit may be hard to test, even with 10 years of Fermi-LAT data, especially if there is a mixture of DM and MSPs contributing to the signal, in which case the implied DM cross section will be suppressed.
\end{abstract}

\section{INTRODUCTION}

Gamma rays constitute an excellent search channel for a signature of pair annihilation of dark matter (DM), since they can propagate almost without absorption from the source to the observer. Amongst all possible target regions in the gamma-ray sky, the Galactic Center is expected to be the brightest DM emitting source as it is relatively close by and has a high density of DM. However, this region is populated by a variety of astrophysical gamma-ray sources that make it hard to unambiguously identify a DM signal 1, 2.

Several independent groups have reported evidence of extended excess gamma-ray emission above the diffuse galactic background (DGB) from the central $1^{\circ}-2^{\circ}$ around the Galactic Center 3 10. These investigation were based on Fermi Large Area Telescope (LAT) data. Although the Fermi-LAT Collaboration have not yet published a full Galactic Center analysis, in a preliminary study with one year of data, the Fermi team has reported an excess in observed counts peaking at energies of $\sim 2-5 \mathrm{GeV}$ [11, 12. Given that there is a reasonable consensus on the reality of these Galactic Center excess gamma rays (GCEG), various alternative explanations for its origin have been posited:

(i) DM particles with masses of about $10-100 \mathrm{GeV}$ annihilating into $b \bar{b}$ and $\tau^{+} \tau^{-}$final states or a combination of both [3, 4, 6, 6]. Importantly, it was argued in Ref. 10] that the signal has a relatively soft spectral shape, which makes it difficult to fit the GCEG data with a dark matter model annihilating mainly to leptons. The spatial profile of the DM was found to be well fit [10] by a generalized NFW profile [13] with inner slope $\gamma=1.2$. As the DM signal is proportional to $\rho^{2}$, the spatial profile used will be the square of a generalized NFW profile with inner slope $\gamma=1.2$. We will denote this spatial profile as $\left(\mathrm{NFW}_{1.2}^{2}\right)$.

(ii) A superposition of $\sim 10^{3}$ millisecond pulsars (MSPs) within a radius of $r \lesssim 150 \mathrm{pc}$ of the Galactic Center whose number density follow a $\mathrm{NFW}_{1.2}^{2}$ profile 8 10, 14 17. However, Refs. [18, 19] have claimed that there is evidence of a gamma-ray excess at $2 \mathrm{kpc} \leq r \leq$ $3 \mathrm{kpc}$ that is consistent with DM annihilation but is too extended to be explained by a concentrated population of MSPs given the number of MSPs that have been resolved by Fermi-LAT 20 .

(iii) Another possibility is that the signal is being produced by cosmic rays interacting with gas in the Galactic Center [3, 6, 8, 9, 21, 22. This alternative solution can be divided in two different scenarios, the hadronic and nonthermal bremsstrahlung. The first one consists of $\pi^{0}$ decays resulting from the emission of high energy protons and their subsequent collision with gas in the Galactic Center. In Ref. [21] it was found that a model based on hadronic emission from Sgr A* would be determined predominately by the gas distribution and would appear point-like to the Femi-LAT gamma-ray detector. Therefore, that model would not be suitable for explaining the extended nature of the GCEG.

In the second scenario, the nonthermal bremsstrahlung emission model, a case which results in extended emission has been proposed in Ref. 22. Based on multiwavelength observational data obtained with the Green Bank Telescope (GBT) [23, Susaku, X-ray Multi-Mirror Mission (XMM)-Newton, Chandra, Fermi-LAT and High Energy Stereoscopic System (HESS) it was argued 22 that the $\sim \mathrm{GeV}$ GCEG is nonthermal, diffuse and is probably generated by a population of synchrotron emitting electrons interacting with gas in molecular clouds.

In this study we focus on the spatial and spectral 
morphology of the gamma-ray Galactic Ridge (hereafter "Galactic Ridge") region, and confirm that an extended source associated with the Galactic Ridge can improve the GCEG fit. But, we find that adding a Galactic Ridge does not remove the need for also adding a spherically symmetric extended source whose radial profile follows a $\mathrm{NFW}_{1.2}^{2}$ profile. We show that the spectral parameters of the $\mathrm{NFW}_{1.2}^{2}$ template are not significantly affected by inclusion of a Galactic Ridge.

\section{DATA REDUCTION}

The Fermi-LAT data selection is the same as described in [10. In summary, we analysed Pass- 们 data taken within a squared region of $7^{\circ} \times 7^{\circ}$ centred on $\mathrm{Sgr} \mathrm{A}^{\star}$ in the first 45 months of observations over the period August 4, 2008-June 6, 2012. We used the standard data cuts and kept only the SOURCE class events which have a high probability of being photons of astrophysical origin. We also selected events between $200 \mathrm{MeV}-100$ $\mathrm{GeV}$ without making any distinction between Front and Back events.

The spectra were obtained by maximizing the likelihood of source models using the binned pyLikelihood library in the Fermi Science Tools [24. We followed the same fitting procedure adopted in Ref. [8, 9] which has been recommended to be more suitable for crowded regions like the Galactic Center. Unless otherwise stated, the models included all sources suggested in the 2FGL 25] catalog plus the LAT standard DGB and extragalactic background models gal_2yearp7v6_v0.fits and iso_p7v6source.txt respectively.

\section{MODELS FOR THE EXTENDED SOURCE AT THE GALACTIC CENTER}

The HESS telescope has revealed a point-source coinciding with the dynamical center of the Milky Way Galaxy as well as diffuse emission that is spatially correlated with the molecular clouds in the Galactic Ridge 26. In Ref. 22 it was argued that bremsstrahlung from nonthermal electrons in Galactic Center molecular clouds can explain the GCEG measured at TeV scales by HESS and at GeV scales by Fermi-LAT. The nonthermal electrons in the molecular clouds are proposed to mainly come from supernova remnants and nonthermal radio filaments (see [22, 23] and references therein). A proposed population of nonthermal electrons is constrained, by both radio and gamma-ray data, to need a broken power law spectrum where the break is attributed

\footnotetext{
${ }^{1}$ Preliminary checks have shown our results are not significantly changed if we instead use Pass-7 reprocessed data.
}

to rapid cooling of electrons at high energies [22. By comparing the frequency of the break in the radio data and the energy of the break in the gamma-ray data, the magnetic field value can be constrained, see Sec. $\mathrm{V}$.

The $\mathrm{TeV}$ nonthermal electrons, proposed to explain for the HESS Galactic Ridge, are assumed to be a separate younger population of nonthermal electrons in the Galactic Center molecular clouds. This extra population is assumed to have not had time to cool and so is modeled with a power law distribution 22 .

To study the evidence for a new component of extended $\mathrm{GeV}$ emission in the Fermi-LAT data, the authors in Ref. 22] tried spatial templates obtained from X-ray, 20$\mathrm{cm}$ continuum emission radio data, and the HESS residuals. For a spectral model they initially employed a broken power law of the form:

$$
\frac{d N}{d E}=N_{0} \times \begin{cases}\left(\frac{E}{E_{b}}\right)^{-\Gamma_{1}} & \text { if } E<E_{b} \\ \left(\frac{E}{E_{b}}\right)^{-\Gamma_{2}} & \text { otherwise. }\end{cases}
$$

They found that the 20-cm radio and HESS residual templates had similar high test statistic (TS) values.

For illustration, we show in Fig. 1 the HESS residual and $20-\mathrm{cm}$ spatial templates. The $20-\mathrm{cm}$ template was based on GBT continuum emission data which measures nonthermal and thermal plasma distributions [22, 23. Note, this is distinct from the 21-cm line temperatures used by the Fermi team in constructing the DGB as that gives a measure of the column density [27. Both templates initially had a DC value, evaluated from a nearby region, subtracted. They have also had Sgr A removed and they have been normalized so that their total area integrated flux is unitary.

To test whether the GCEG is better fitted by a combination of a $\mathrm{NFW}_{1.2}^{2}$ template and a Galactic Ridge template we have done a broad band analysis within the Fermi Tools and also a bin-by-bin analysis for each of the extended sources under scrutiny.

\section{SYSTEMATIC ERRORS AND PARAMETER CONSTRAINTS}

The DGB accounts for a large proportion of the photons detected by the LAT instrument. For regions near the Galactic Center this component can be several orders of magnitude brighter than any other source. In particular, the dominant systematic error at energies $\sim 1 \mathrm{GeV}$ emerges from the uncertainties in the DGB model. These systematics were studied in a previous analysis [10.

Since this work involves the analysis of an extra extended source (see section III) not considered in [10, we have reassessed the systematic errors in the DGB by following the same approach explained in 10 . There is consistency between the present and previous analysis [10, we found that the overall systematic flux error is energy 

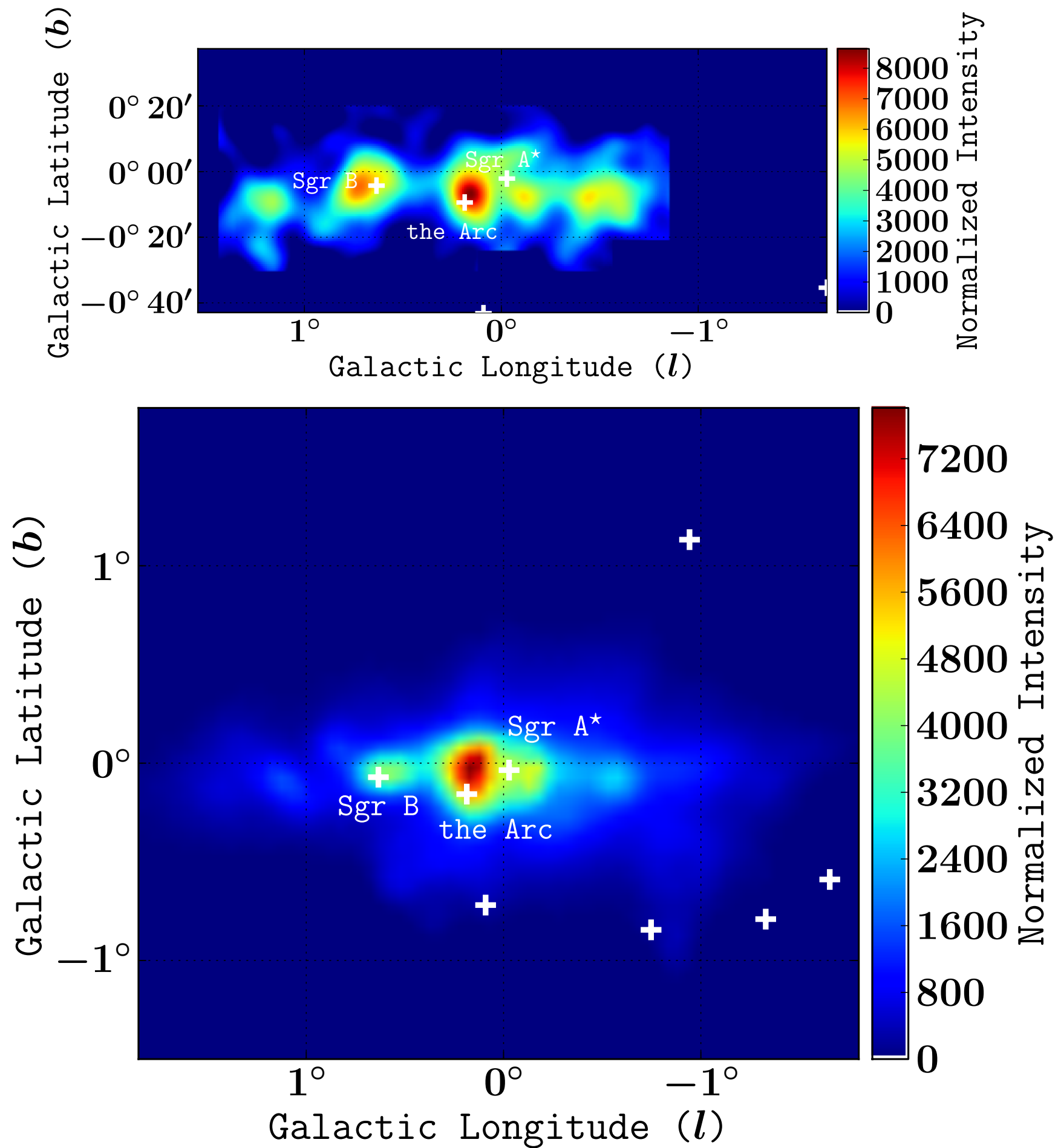

FIG. 1. Top: Gamma-ray image of the Galactic Center as observed by the HESS telescope $(E>380 \mathrm{GeV})$ after subtracting the dominant point sources [26]. To include this template map in the likelihood function within the Fermi Science Tools package, we background subtracted, thresholded and normalized the data provided in [26]. Bottom: Background-subtracted, thresholded, and normalized image from $20-\mathrm{cm}$ continuum emission GBT data. This template was the same one as used in Ref. [22] and we refer the reader to that article for detaill " This spatial template is named the "20-cm template" in the rest of this work. The crosses overlaid on the image represent the position of the 2FGL catalog point sources.

${ }^{\text {a }}$ We thank Farhad Yusef-Zadeh for providing us with the 20-cm template. 
and spatial dependent: systematic errors due to uncertainties of the spectral distribution amounts to an average of about $2 \%$ at $\sim 1 \mathrm{GeV}$, and the dominant fraction for the systematics arises from the spatial part, we obtained on average about $23 \%$ for energy bins $\leq 10 \mathrm{GeV}$ and $18 \%$ in the 10-100 GeV energy band. The total systematic error is evaluated by summing in quadrature the spatial, spectral, and effective area [10, 25] systematic errors.

Our parameter constraints method is the same as used in Ref. [10. In summary we use the Fermi Tools to construct a spectrum of the source of interest [25. As in Refs. [10, 25], we allow the amplitude of all sources, in the region of interest, to vary when fitting a band. We then add, in quadrature, the systematic errors (evaluated as described above) to the statistical errors of the spectral bands. The spectrum likelihood is then approximated as a multivariate Gaussian and a profile likelihood approach is used to construct confidence intervals.

In plotting the spectra we display both the systematic and statistical errors. For bands which have a test statistic (TS) [10, 25] less than 10, or whose total error is more than the half of the best fit band value, we plot the $95 \%$ upper limit. We do not plot or use bands in our parameter constraints which have TS $<1$. Unless otherwise stated, best fit parameter values are quoted with $68 \%$ confidence intervals.

To cross-check the systematical errors explained above, we have also estimated the systematic uncertainties in the DGB following the interesting analysis technique utilized in 28. We constructed eight different diffuse emission models using GALPROP [29, 30, and each of these templates were included in the likelihood fit of the sources of interest as an alternative to the standard DGB recommended in the 2FGL catalog [25].

The set of alternative DGB models taken into account in this analysis consider a range of possible values for the input parameters that were found to exhibit the largest sensitivities [28]. The parameters varied in the models are the cosmic ray propagation halo heights $(4 \mathrm{kpc}$ or 10 $\mathrm{kpc}$ ), cosmic ray source distribution (supernova remnants or pulsars) and the atomic hydrogen spin temperature (150 K or optically thin). An $E(B-V)$ magnitude cut of $5 \mathrm{mag}$ was also chosen. The results obtained through this method are displayed as grey shaded areas in the spectra of Fig. 2.

The DGB provided with the Fermi Tools is generated by a weighted sum of gas column densities and an inverse Compton intensity map [27]. In the DGB generation, the weights depend on the energy band and the gas template weights also depend on the ring radius concentric around the Galactic Center. The weights are fitted to all sky Fermi-LAT data. Due to the greater degree of freedom this method produces a better fit to the Fermi-LAT data than the GALPROP based approach described above. So in general the GALPROP simulations do not envelope the solution found using the standard DGB. Therefore we use the relative dispersion of the GALPROP simulations in constructing the grey bands in the top panels of Fig 2 , In the bottom panel of Fig 2 we plot the band of solutions obtained when the GALPROP DGB's are used. In this panel, the $\mathrm{NFW}_{1.2}^{2}$ template has a greater amplitude as the GALPROP estimate of the DGB is not as good a fit as the standard DGB provided with the Fermi Tools.

\section{RESULTS}

As seen from Fig. 1, the Arc and Sgr B are bright sources in the Galactic Center. They are thought to be associated with cosmic rays interacting with molecular clouds [22] and so in Table I] we consider models with and without them being assumed to be included in the Galactic Ridge template.

The results listed in Table $\llbracket$ show that the broad band analysis revealed significant detections of both a Galactic Ridge and a $\mathrm{NFW}_{1.2}^{2}$ extended source.

The need for the Galactic Ridge can be seen in the residuals shown in Fig. 3. It is particularly noticeable in those bands which have a high TS (see TableVI).

Based on the GBT radio data, Ref. 22] set the synchrotron flux at $325 \mathrm{MHz}$ to be $F_{325}=508 \mathrm{Jy}$ and a synchrotron spectrum of electrons of the form $E^{-p}$ with $p=1.5$ below the break frequency $\nu_{b}=3.3 \mathrm{GHz}$ and $p=4.4$ above it. The GCEG spectrum can be used to constrain the break energy for the electron spectrum $\left(E_{b}\right)$ via Eq. A16. This can be converted to a constraint on the magnetic field strength $B$ by using the measured radio frequency spectral break $\nu_{b}$ and the general relation between electron energy and characteristic synchrotron radio frequency given in Eq. A7. The GBT uncertainties for the spectral slopes, $\nu_{b}$, and $F_{325}$ were not given in Ref. 22] and so our analysis just includes their point estimates.

Fitting the bremsstrahlung model (Eq. A16), we varied the number density of hydrogen nuclei $n_{H}$ and the magnetic field $B$. We simultaneously fit the normalization and slope of the power-law formula corresponding to the TeV HESS data. Using a bin-by-bin analysis we made a parameter scan as shown in Fig. 4 and Table II.

Additionally, this analysis enabled us to study to what extent the Galactic Ridge component affects the model parameters of a DM or unresolved MSPs extended source. We therefore made a detailed parameter scan corresponding to the DM and MSPs hypotheses in models which included a Galactic Ridge. The dark matter spectra are obtained using DMFIT [31] while the standard exponential cut off form is used for the MSPs' spectrum:

$$
\frac{d N}{d E}=K\left(\frac{E}{E_{0}}\right)^{-\Gamma} \exp \left(-\frac{E}{E_{\text {cut }}}\right)
$$

where photon index $\Gamma$, a cut-off energy $E_{\text {cut }}$ and a normalization factor $K$ are free parameters. The results are summarized in Fig. 5 and Table III for the MSPs hypothesis, and Fig. 6 and Table IV for the DM hypothesis. 

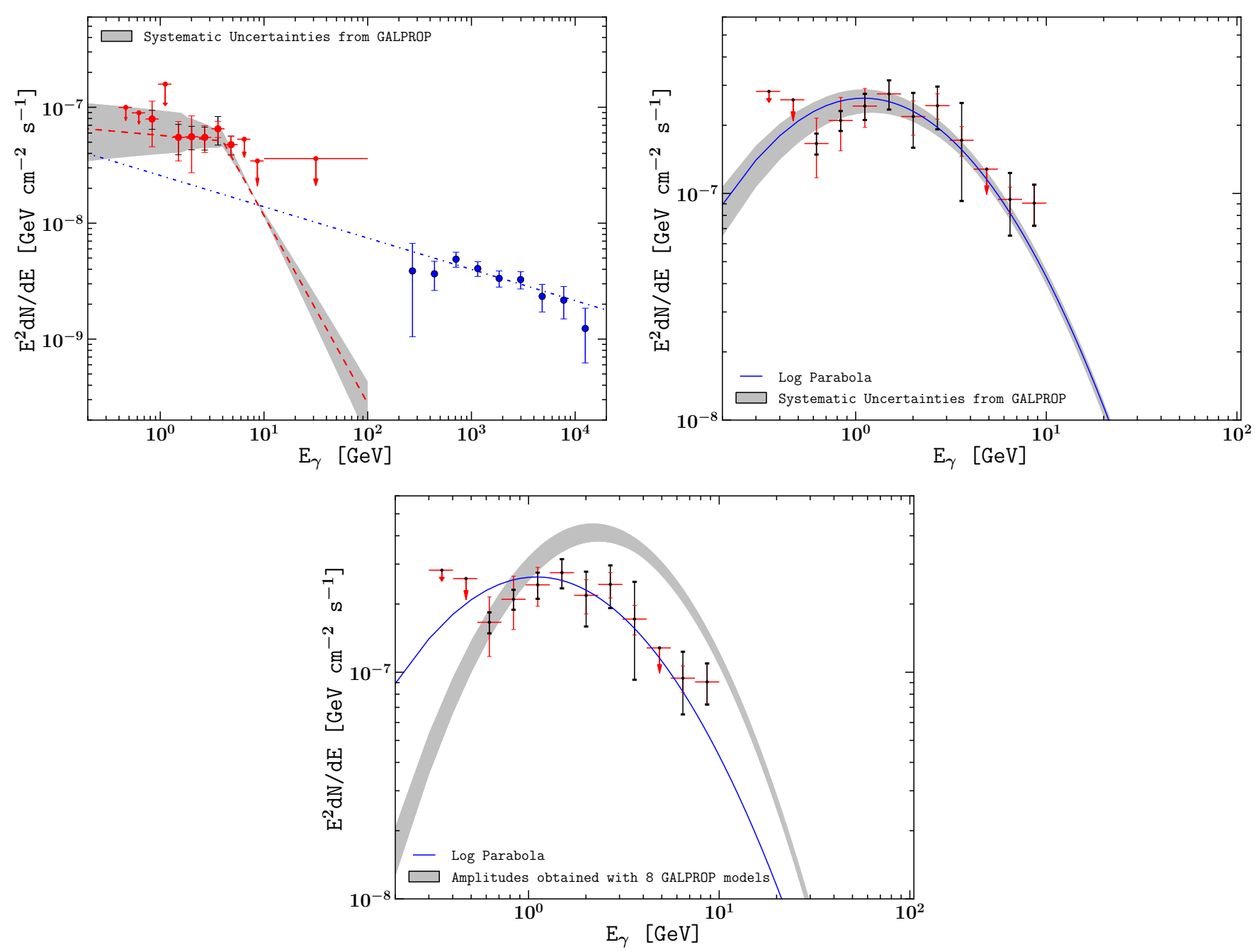

FIG. 2. Top Left: Galactic Ridge spectrum generated from model 2 in Table I The red dashed line shows the best fit broken power-law as obtained from a Fermi Tools broad band fit. The grey area is an estimate of the systematic uncertainties as calculated with 8 different GALPROP models of the DGB. Black and red error bars are the LAT $(1 \sigma)$ statistical and systematic errors. A red arrow indicates a 95\% upper limit. The blue points correspond to data taken by HESS 26] and the blue dotted line is the best fit power law to them. Top Right: spectrum for $\mathrm{NFW}_{1.2}^{2}$ spatial profile generated with model 1 in Table I. The blue solid line is the best fit Fermi tools log parabola spectrum. The grey area shows the systematic uncertainties as computed with 8 different GALPROP models of the DGB. The spectra and error bars are listed in Table V]and VI Bottom Center: Same as top right, except the GALPROP based model results are shown rather than just the relative errors obtained from them.

\section{DISCUSSION}

The main focus of our study was try to evaluate three competing explanations for the GCEG: MSPs, DM, or a Galactic Ridge resulting from the interaction of cosmic rays with molecular clouds. As we discuss below, the data prefer combinations of the Galactic Ridge template and a $\mathrm{NFW}_{1.2}^{2}$ template which has a spectrum compatible with either MSPs, DM, or some combination of the two.

\section{A. Interaction of cosmic rays with molecular clouds}

From Table $\mathrm{I}$ we can check the significance of adding a new component by evaluating the test statistic (TS) which is defined as in Ref. 25]:

$\mathrm{TS}=2[\log \mathcal{L}($ new source $)-\log \mathcal{L}($ NO-new source $)]$,

where $\mathcal{L}$ stands for the maximum of the likelihood of the data given the model with or without the new source. In the large sample limit, under the no source hypothesis, TS has a $\chi^{2} / 2$ distribution with the number of degrees of freedom equal to the number of parameters associated with the proposed positive amplitude new source [36, 37]. 

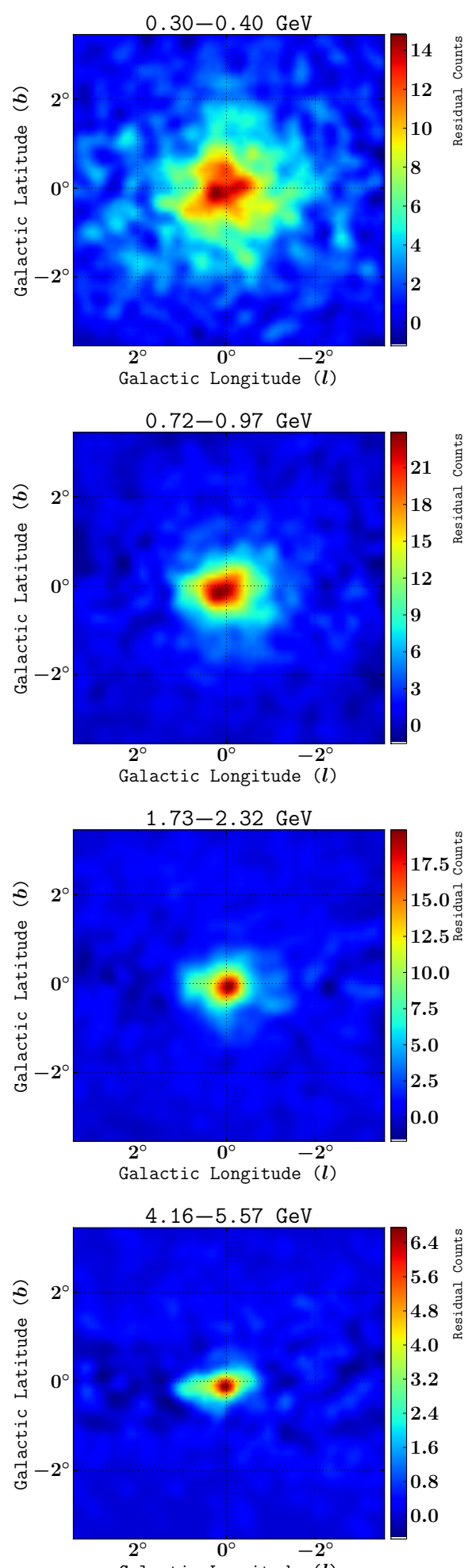

Galactic Longitude $(l)$
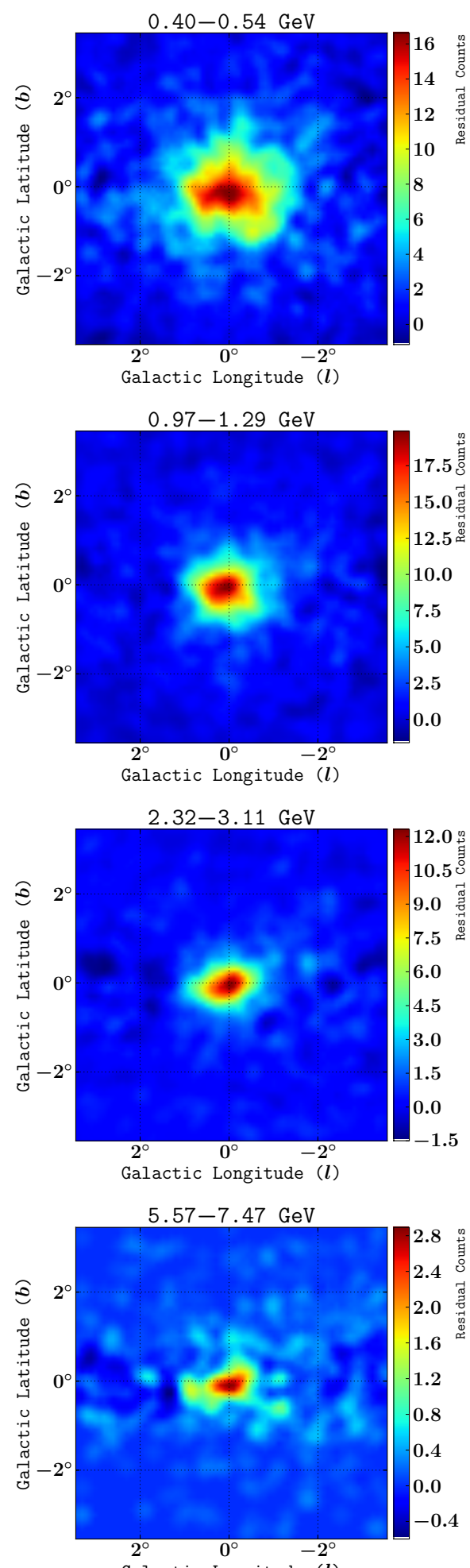

Galactic Longitude $(l)$
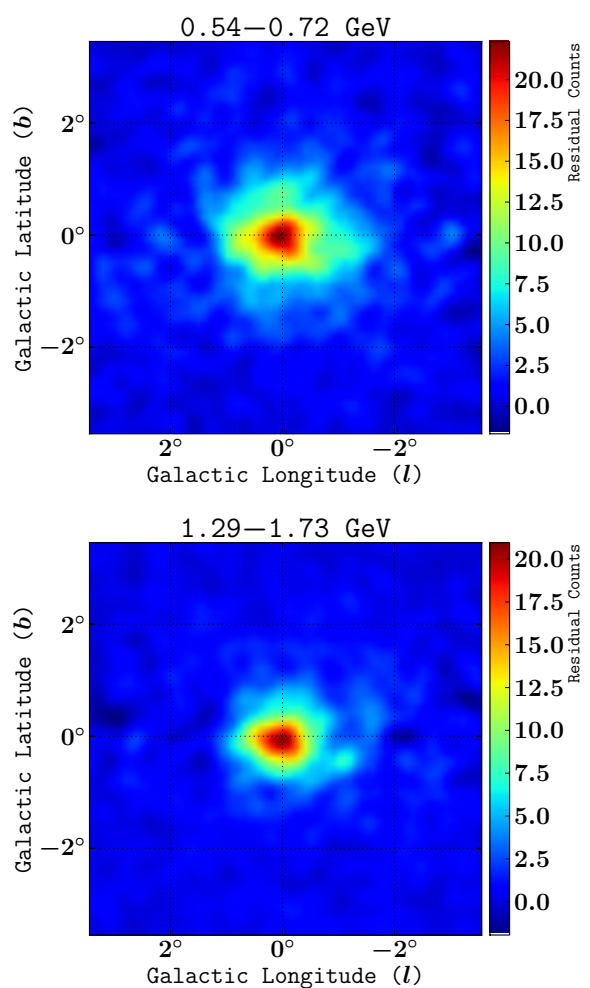

$3.11-4.16 \mathrm{GeV}$

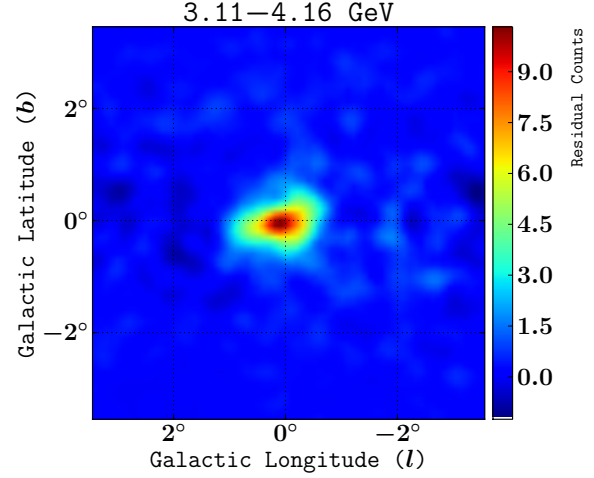

$7.47-10.00 \mathrm{GeV}$

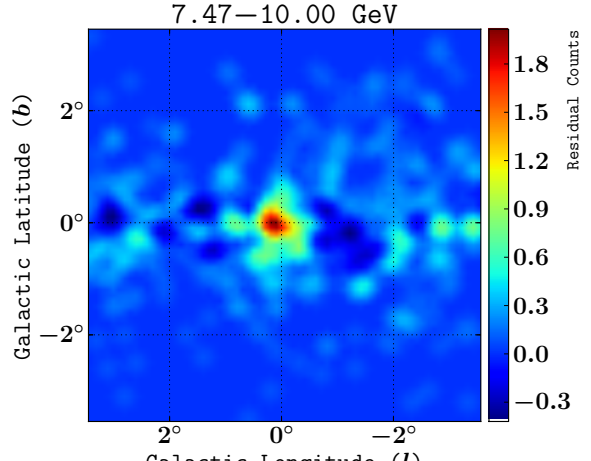

Galactic Longitude $(l)$

FIG. 3. Shown are the residuals of model 2 in Table $\mathrm{I}$ where the model components of the NFW $\mathrm{N}_{1.2}^{2}$ and the 20 -cm Galactic Ridge have not been subtracted from the data. The images have been smoothed with a $0.3^{\circ}$ radius Gaussian filter. 


\begin{tabular}{|c|c|c|}
\hline Model & $2 \log \left(\mathcal{L} / \mathcal{L}_{\text {base }}\right)$ & dof-dof $f_{\text {base }}$ \\
\hline Base (2FGL-"the Arc"-Sgr B) & 0 & 0 \\
\hline $2 \mathrm{FGL}$ & 425 & $4+5=9$ \\
\hline $2 \mathrm{FGL}+20-\mathrm{cm}$ template & 638 & $4+5+4=13$ \\
\hline $2 \mathrm{FGL}+\mathrm{NFW}_{1.2}^{2}$ & 1295 & $4+5+3=12$ \\
\hline $2 \mathrm{FGL}+\mathrm{NFW}_{1.2}^{2}+$ HESS residual template & 1325 & $4+5+3+4=16$ \\
\hline $2 \mathrm{FGL}+\mathrm{NFW}_{1.2}^{2}+20-\mathrm{cm}$ template $(\operatorname{model} \mathbf{1})$ & 1330 & $4+5+3+4=16$ \\
\hline Base+NFW ${ }_{1.2}^{2}+$ HESS residual template & 1164 & $3+4=7$ \\
\hline Base $+\mathrm{NFW}_{1.2}^{2}+20-\mathrm{cm}$ template $(\operatorname{model} 2)$ & 1170 & $3+4=7$ \\
\hline
\end{tabular}

TABLE I. The likelihoods evaluated in compiling the above table are maximized with a broad band analysis using the Fermi Tools. Alternatives models of the Galactic Center in the $200 \mathrm{MeV}-100 \mathrm{GeV}$ energy range are listed. Each point source in the model has degrees of freedom (dof) from its spectrum and two extra dof from its location. The spectra for the Galactic Ridge templates are modeled by a broken power law. While the spectra for the $\mathrm{NFW}_{1.2}^{2}$ templates are modeled by a log parabola which has enough flexibility to mimic a good fitting DM or MSP spectra 10].
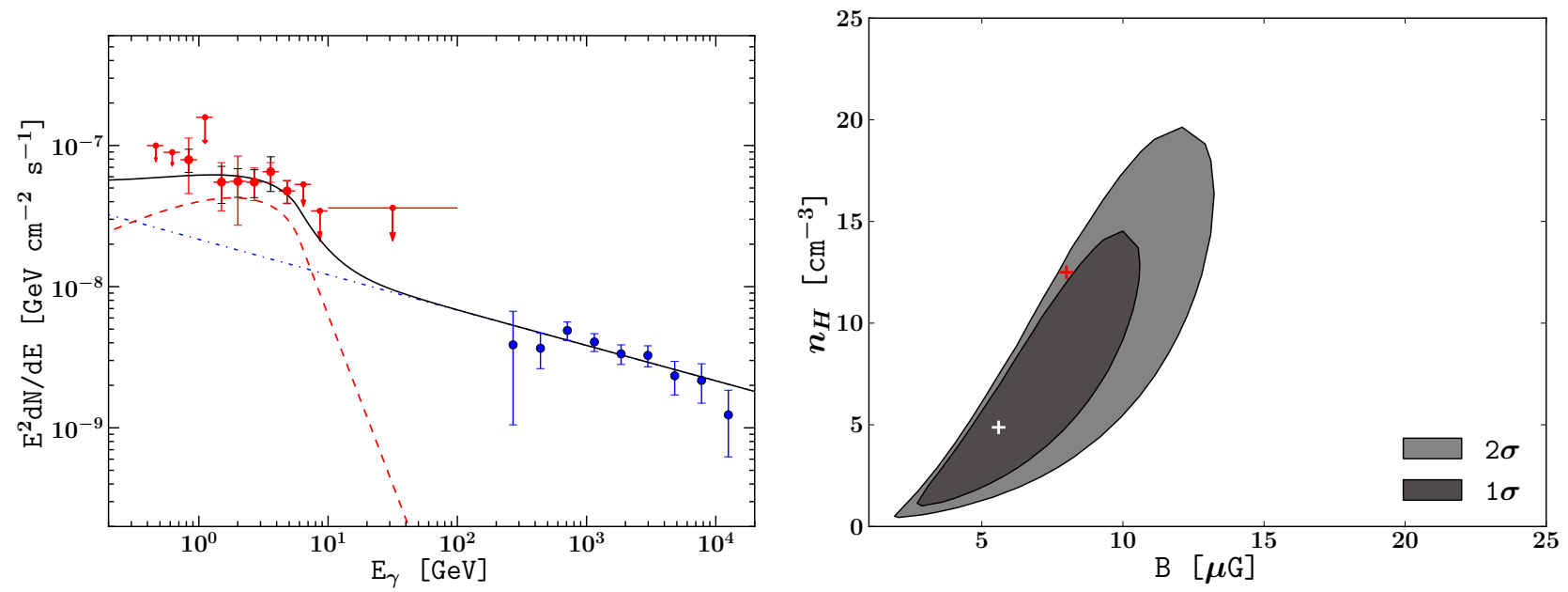

FIG. 4. (Left) Red filled circles show the Fermi-LAT Galactic Ridge energy flux points obtained under the assumption of Model 2 in Table II They are listed in Table VI The blue circles represent the Galactic Ridge as measured by the HESS telescope [26]. Black and red error bars show statistical and systematic errors respectively. Red arrows show $2 \sigma$ upper limits. The red dashed curve is the gamma-ray nonthermal bremsstrahlung model generated from Eq. A16 The blue dotted line is a nonthermal bremsstrahlung model represented by a power law. The black solid line is the sum of the red dashed curve and blue dotted line. It gives the best fit to the combined Fermi-LAT and HESS Galactic Ridge data. (Right) Confidence regions generated from the data and models shown in the left panel. The parameter $n_{H}$ is the number density of hydrogen nuclei and $B$ the magnetic field. The white cross shows our best-fit values while the red cross corresponds to the values found in Ref. 22 . See also Table II

\begin{tabular}{lccc}
\hline \hline$n_{\mathrm{H}}\left[\mathrm{cm}^{-3}\right]$ & $B[\mu \mathrm{G}]$ & $N_{0}\left[\mathrm{ph} \mathrm{cm}^{-2} \mathrm{~s}^{-1} \mathrm{MeV}^{-1}\right]$ & $\Gamma$ \\
\hline $5_{-3}^{+6}$ & $6_{-2}^{+3}$ & $(2 \pm 1) \times 10^{-11}$ & $2.25_{-0.08}^{+0.07}$ \\
\hline \hline
\end{tabular}

TABLE II. Best fit values obtained in the bremsstrahlung analysis for the gas number density $\left(n_{\mathrm{H}}\right)$, the magnetic field $(B)$, and the HESS power law spectrum amplitude $\left(N_{0}\right)$ and spectral index $(\Gamma)$. The best fit spectra and data fitted to are shown in the LHS panel of Fig. 4

As the amplitude is restricted to be non-negative, a $\chi^{2} / 2$ distribution rather than the $\chi^{2}$ distribution is needed.

As can be seen from Table I] the improvement in the fit of the 20-cm Galactic Ridge relative to 2FGL is TS= $648-425=213$ for $13-9=4$ extra degrees of freedom (dof). This corresponds to a $14 \sigma$ detection (if we convert to the equivalent p-value for 1 degree of freedom) and so confirms that the 20-cm Galactic Ridge does improve the fit to the GCEG. However, the corresponding TS for a $\mathrm{NFW}_{1.2}^{2}$ template is 870 and for only 3 extra dof and so clearly also improves the fit substantially.

We can check whether the 20-cm Galactic Ridge still 


\begin{tabular}{lccc}
\hline \hline Model & $E_{\text {cut }}[\mathrm{GeV}]$ & $\Gamma$ & $G_{100}\left[10^{-9} \mathrm{erg} \mathrm{cm}^{-2} \mathrm{~s}^{-1}\right]$ \\
\hline MSPs & $4_{-1}^{+2}$ & $1.6 \pm 0.2$ & $1.5 \pm 0.2$ \\
MSPs + Galactic Ridge & $3_{-1}^{+2}$ & $1.4 \pm 0.3$ & $1.2_{-0.1}^{+0.2}$ \\
\hline \hline
\end{tabular}

TABLE III. Best-fit values for MSPs hypothesis. The spectrum of the MSPs is fitted with a power law with an exponential cut off (see Fig. 5). The first row shows the result from an analysis without a galactic ridge 10 . The second row parameters were fitted to the spectral data plotted on the top RHS panel of Fig. 2. The GCEG energy flux for $100 \mathrm{MeV} \leq E \leq 100 \mathrm{GeV}$ is denoted by $G_{100}$.

\begin{tabular}{lccc}
\hline \hline Model & Best-fit Branching ratio & $\langle\sigma v\rangle\left[\mathrm{cm}^{3} / \mathrm{s}\right]$ & $M_{\mathrm{DM}}[\mathrm{GeV}]$ \\
\hline $\mathrm{DM}$ & $(60 \pm 20) \% b \bar{b}$ & $(2.8 \pm 0.4) \times 10^{-26}$ & $24 \pm 7$ \\
$\mathrm{DM}+20-\mathrm{cm}$ template & $(80 \pm 20) \% b \bar{b}$ & $2.0_{-0.6}^{+0.5} \times 10^{-26}$ & $27_{-9}^{+8}$ \\
\hline \hline
\end{tabular}

TABLE IV. Best-fit values for the branching fraction between $b \bar{b}$ and $\tau^{+} \tau^{-}$, DM velocity averaged annihilation cross section and DM mass. DMFIT was used to generate the model spectra 31. The first row shows the result from an analysis without a Galactic Ridge [10]. The second row parameters were fitted to the spectral data plotted on the top RHS panel of Fig. 2 .

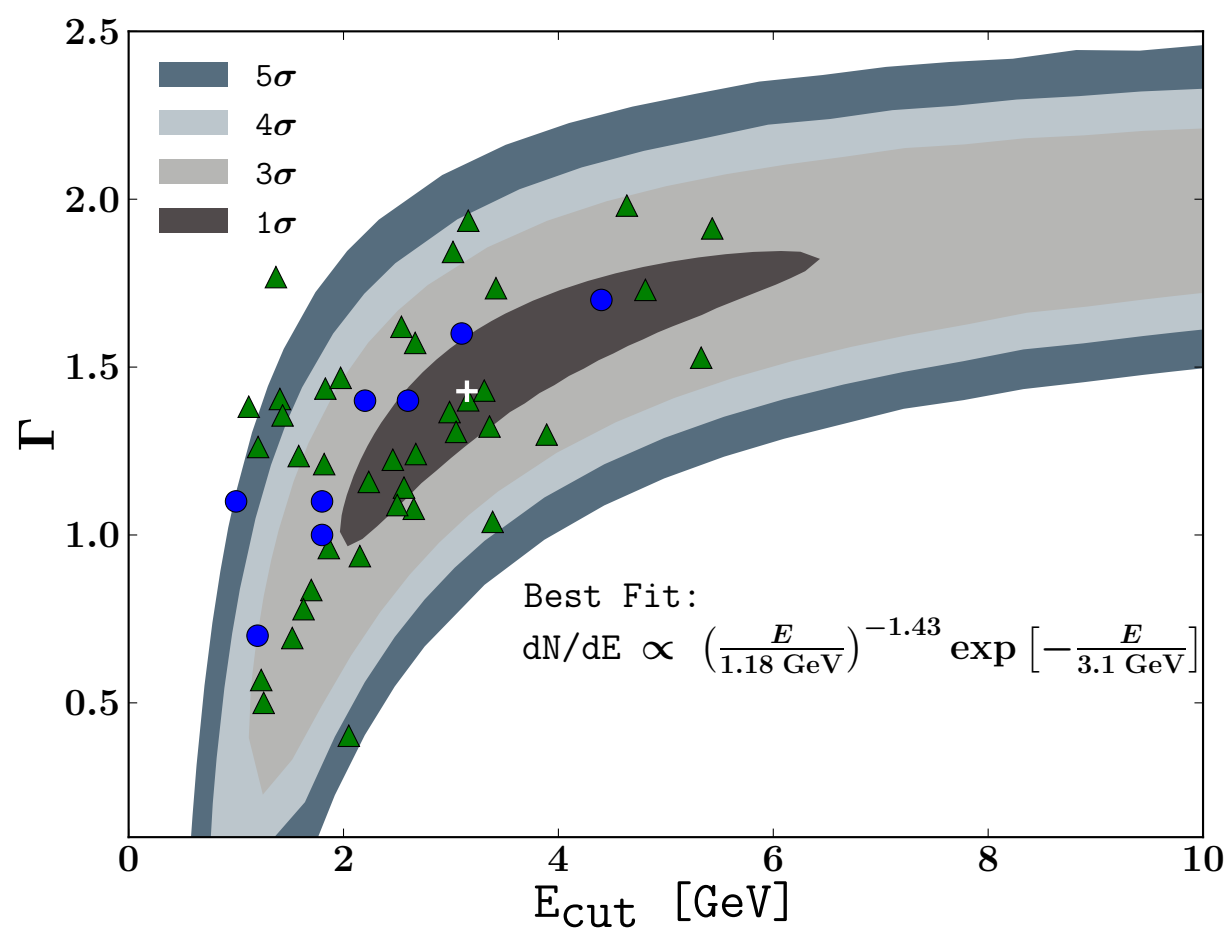

FIG. 5. Confidence regions for an unresolved population of MSPs when the Galactic Ridge was included in the fit. The data used is shown in the top RHS panel of Fig. 2 and listed in Table V. The best fit is denoted by a white cross. The green triangles show the best fit parameters of the MSPs detected in the second Fermi LAT catalog of gamma-ray pulsars (2FPC) 32. The blue circles represent the best fit parameters of MSP populated globular clusters [33.

improves the fit once the $\mathrm{NFW}_{1.2}^{2}$ template is included. From Table 【 we obtain a TS= $1330-1295=35$ for 4 extra dof which corresponds to a $5 \sigma$ detection. This shows that the GCEG motivates a sum of the $\mathrm{NFW}_{1.2}^{2}$ and the Galactic Ridge being included.

The parts of the data which require the $\mathrm{NFW}_{1.2}^{2}$ and the 20-cm Galactic Ridge are shown in Fig. 3. The elon- gation in the longitudinal direction, indicating the need for the 20-cm Galactic Ridge, is particularly evident in the energy ranges 1.73 to $5.57 \mathrm{GeV}$.

We also did the above analysis with the HESS residual Galactic Ridge and we found that a TS=30 for 4 extra dof which is less than the $20-\mathrm{cm}$ case, but the difference is not statistically significant. 

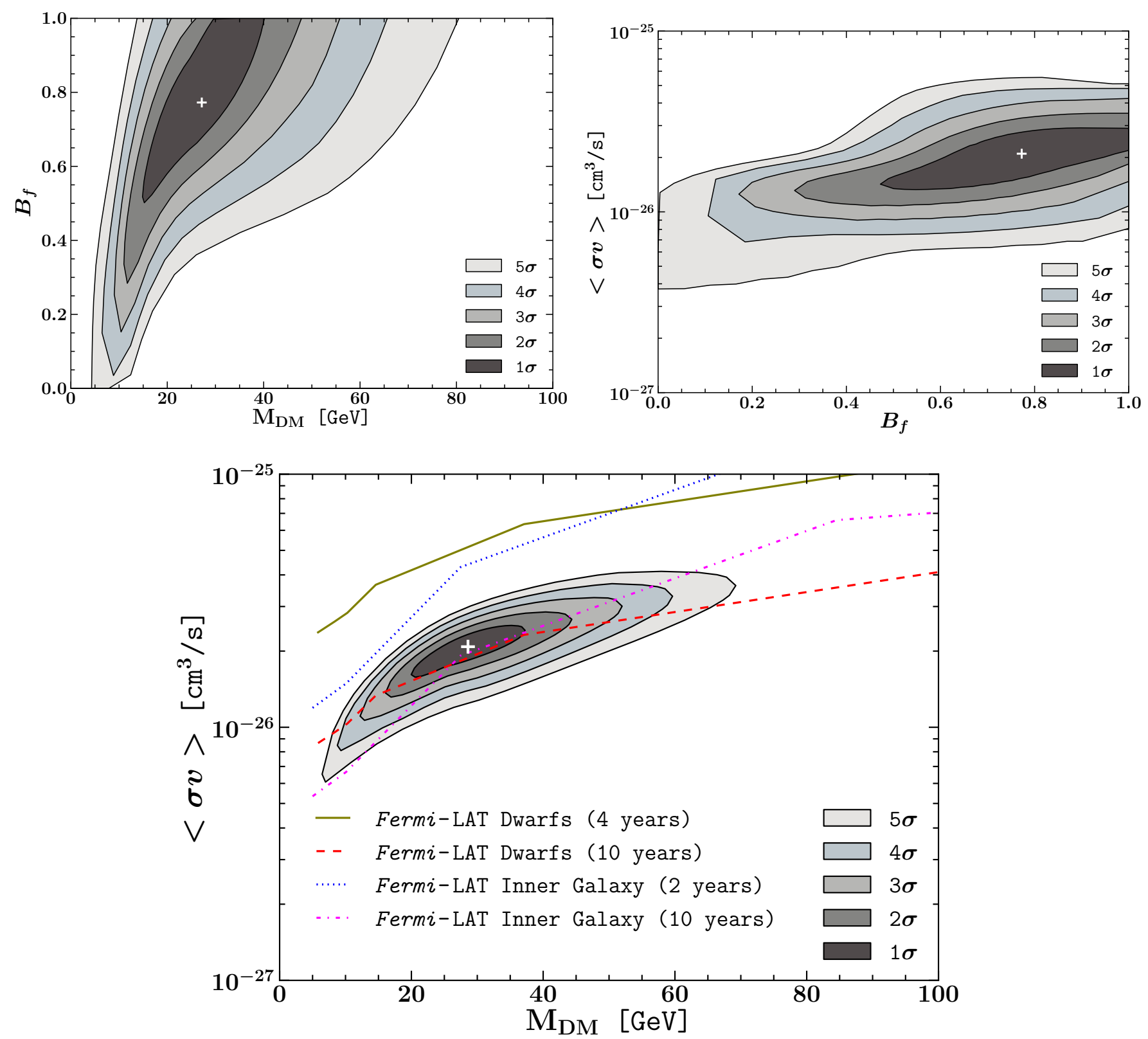

FIG. 6. Confidence regions for the dark matter model when the Galactic Ridge source was included. The data used is shown in the top RHS panel of Fig. 2 and listed in Table V] The parameter $B_{f}=1.0$ implies $100 \% b \bar{b}$ and $B_{f}=0.0$ means $100 \%$ $\tau^{+} \tau^{-}$. The DM spatial distribution follows a NFW profile with inner slope $\gamma=1.2$. The white cross denotes the best-fit values. Limits from dwarf galaxies [34] $(2 \sigma)$ and the Inner Galaxy $\left(10^{\circ} \leq b \leq 20^{\circ}\right)(3 \sigma)$ 35] are included. We rescaled the Inner Galaxy results to account for the different $\gamma$ of the current study, using the Galactic coordinate $(l=0, b=10)$ as a reference point 10 . The 10 year forecasts were approximated with a simple $1 / \sqrt{\text { time }}$ scaling and in the dwarf galaxy case it was assumed there were three times more dwarf galaxies available.

Additionally, we checked whether the inclusion of the Galactic Ridge affected the spectral parameters of the $\mathrm{NFW}_{1.2}^{2}$ model. As can be seen from Tables III and IV, the inclusion of the Galactic Ridge does not significantly alter the spectral parameters of the $\mathrm{NFW}_{1.2}^{2}$ template.

In Ref. 22], it was argued that the Arc and Sgr B were associated with cosmic rays interacting with molecular clouds and so should not be included when evaluating the parameters of the Galactic Ridge. They also investigated the effects of adding Sgr C, but we found once the $\mathrm{NFW}_{1.2}^{2}$ was included, Sgr C had a very low TS and so we have not included it in our analysis.

As can be seen from Table I the Arc and Sgr B do significantly improve the fit even when the Galactic Ridge and the $\mathrm{NFW}_{1.2}^{2}$ template are included. However, it is common practice [38, 39] to exclude such point sources 
when analyzing a physical model for the cosmic rays interacting with molecular gas. Otherwise, some of the signal will be lost to the apparent point sources arising from cosmic rays interacting with molecular gas. As shown in Table I] in this case there is also no significant difference in the goodness of fit between HESS Galactic Ridge and 20-cm Galactic Ridge, but as the 20-cm template has a slightly higher TS in both models 1 and 2, we use it as the default.

In Fig. 4we provide confidence regions for the magnetic field $B$ and hydrogen density $n_{H}$. The Galactic Ridge is consistent with the Ref. [22] best fit even though the extra $\mathrm{NFW}_{1.2}^{2}$ component is included.

\section{B. Millisecond Pulsars}

In Fig. 5 we show the confidence intervals for the exponential cut off fit. Although, they are not significantly different from the ones without an extended Galactic Ridge template (see Ref.[10]), here we also show the MSPs reported in the second Fermi LAT catalog of gamma-ray pulsars (2FPC) 32 and also the globular clusters which can contain multiple unresolved MSPs 33. As can be seen the spectrum of the GCEG is consistent with the majority of MSPs and MSP containing globular clusters.

Using the GCEG energy flux for $100 \mathrm{MeV} \leq E \leq 100$ $\mathrm{GeV}\left(G_{100}\right)$ of our best fit exponential cut off model from Table III we evaluate the luminosity as $L_{\gamma}=4 \pi d^{2} G_{100} \sim$ $10^{37} \mathrm{erg} \mathrm{s}^{-1}$ where we take the distance to the Galactic Center as $d \sim 8 \mathrm{kpc}$.

The 2FPC contains 40 MSPs with estimated luminosities ranging from about $5 \times 10^{31}$ to $7 \times 10^{34} \mathrm{erg} \mathrm{s}^{-1}$. The average MSP luminosity in the $2 \mathrm{FPC}$ is $\bar{L}_{\mathrm{MSP}} \sim 10^{34} \mathrm{erg}$ $\mathrm{s}^{-1}$. Only about $20 \%$ of known MSPs have been detected by Fermi-LAT 32 and so the catalog is biased towards higher gamma-ray luminosity MSPs. Therefore, we expect the $2 \mathrm{FPC}$ average MSP luminosity will be greater than the MSP population average. So we use the average $2 \mathrm{FPC}$ value to estimate a lower bound of $\sim 1000 \mathrm{MSPs}$ for $r \lesssim 150$ pc in order to explain the GCEG.

If we assume each of the MSPs at the Galactic Center has a luminosity of $\bar{L}_{\mathrm{MSP}}$ and then convert this to a flux using $d \sim 8 \mathrm{kpc}$ we get each MSP at the Galactic Center has a flux $\sim 10^{-12} \mathrm{erg} \mathrm{cm}^{-2} \mathrm{~s}^{-1}$ which, as can be seen from Fig. 17 of Ref. 32, is below the detection limit $\left(\sim 10^{-11}\right.$ erg $\left.\mathrm{cm}^{-2} \mathrm{~s}^{-1}\right)$ at the Galactic Center. This is consistent with these proposed Galactic Center MSPs being unresolved.

The Galactic Center $r \lesssim 150$ pc region corresponds to about 6 square degrees. As the Fermi-LAT resolution is $\gtrsim 0.1^{\circ}$ at the relevant energy level of this work, it follows that each $(0.1 \mathrm{deg})^{2}$ pixel of the Fermi-LAT image of the Galactic Center would contain $\gtrsim 1 \mathrm{MSP}$.

This MSPs explanation of the GCEG is consistent with the results presented in Ref. [15. Their analysis is based on the number of neutron stars which are computed from the core collapse supernovae rate which in turn is obtained from measurements of the total mass of ${ }^{26} \mathrm{Al}$ in the Galaxy. Using this method they estimate the number of MSPs as $\sim 10^{5} f_{r}$ for $r \lesssim 150$ pc where $f_{r}$ is the fraction of neutron stars that get recycled to MSPs. Based on Galactic disk and globular cluster radio observations, they estimate $f_{r} \sim 10^{-2}$ for $r \lesssim 150 \mathrm{pc}$.

A justification for the MSPs resulting in a $\mathrm{NFW}_{1.2}^{2}$ profile with $\gamma \sim 1.2$ was proposed in Ref. 8 who noted the observations of low mass X-ray binaries (LMXBs) in M31 follow a similar profile and MSPs are believed to arise from LMXBs. They also note some indication of a similar trend in the Milky Way although the LMXB observational data in that case is currently not very conclusive.

\section{Dark Matter}

Although the estimates for the DM parameters are not significantly changed, as can be seen from Fig. 6, the $\tau^{+} \tau^{-}$channel is now only excluded as $4 \sigma$ rather than $5 \sigma$ as was the case when no Galactic Ridge was included 10. As can be seen in the bottom panel of Fig. 6, neither Fermi-LAT dwarfs nor the Fermi-LAT inner Galaxy will be able to definitively detect the DM self-annihilation if it is causing the GCEG. Also, as there is likely to be at least some MSP contribution, the actual $\langle\sigma v\rangle$ will be correspondingly lower and so even harder to detect.

\section{CONCLUSIONS}

We have found that the GCEG is best fit by adding to the base $2 \mathrm{FGL}$ model both a $\mathrm{NFW}_{1.2}^{2}$ source and a Galactic Ridge based on a 20-cm continuum emission template. Similar results were found for a Galactic Ridge template based on the HESS data residuals. The addition of the Galactic Ridge was not found to significantly affect the $\mathrm{NFW}_{1.2}^{2}$ spectral parameters. We found that the GCEG is consistent with a lower bound of $\sim 1000$ on the number of MSPs at the Galactic Center. This is consistent with estimates based on core collapse supernovae inferences from ${ }^{26} \mathrm{Al}$ measurements. We also demonstrated that current and 10-year Fermi-LAT measurements of dwarf spheroidals and the inner Galaxy are unlikely to be able to conclusively check a DM annihilation explanation of the GCEG. As the modeling based on the ${ }^{26} \mathrm{Al}$ measurements indicates there is likely to be $\sim 1000$ MSPs in the Galactic Center, this implies that if there is a DM annihilation component then it probably has a significantly smaller $\langle\sigma v\rangle$ and so will be even harder to check.

We also constrained a bremsstrahlung model of the Galactic Ridge and showed that the $B$ and $n_{H}$ constraints are consistent with a previous analysis [22] done without a $\mathrm{NFW}_{1.2}^{2}$ component. 


\section{ACKNOWLEDGMENTS}

We thank Mark Wardle for providing notes for the bremsstrahlung formula used in the analysis of Ref. [22]. We also thank Farhad Yusef-Zadeh for providing the 20- cm template and helpful comments on the underlying physics it measures. O.M. is supported by a UC Doctoral Scholarship. This work makes use of FERMI SCIence Tools 24], DMFit [31, Minuit 40, SciPy 41], and GALPROP [29, 30].
[1] Marco Cirelli, "Indirect Searches for Dark Matter: a status review," (2012), arXiv:1202.1454 [hep-ph].

[2] Stefan Funk, "Indirect Detection of Dark Matter with gamma rays," (2013), arXiv:1310.2695 [astro-ph.HE].

[3] Lisa Goodenough and Dan Hooper, "Possible Evidence For Dark Matter Annihilation In The Inner Milky Way From The Fermi Gamma Ray Space Telescope," (2009), arXiv:0910.2998 [hep-ph].

[4] D. Hooper and L. Goodenough, "Dark Matter Annihilation in The Galactic Center As Seen by the Fermi Gamma Ray Space Telescope," Phys.Lett. B697, 412-428 (2011), arXiv:1010.2752 [hep-ph]

[5] Alexey Boyarsky, Denys Malyshev, and Oleg Ruchayskiy, "A comment on the emission from the Galactic Center as seen by the Fermi telescope," Phys.Lett. B705, 165-169 (2011), arXiv:1012.5839 [hep-ph].

[6] D. Hooper and T. Linden, "On The Origin Of The Gamma Rays From The Galactic Center," Phys.Rev. D84, 123005 (2011), arXiv:1110.0006 [astro-ph.HE]

[7] Dan Hooper, Chris Kelso, and Farinaldo S. Queiroz, "Stringent and Robust Constraints on the Dark Matter Annihilation Cross Section From the Region of the Galactic Center," (2012), arXiv:1209.3015 [astro-ph.HE]

[8] Kevork N. Abazajian and Manoj Kaplinghat, "Detection of a Gamma-Ray Source in the Galactic Center Consistent with Extended Emission from Dark Matter Annihilation and Concentrated Astrophysical Emission," Phys.Rev. D86, 083511 (2012), arXiv:1207.6047 [astroph.HE]

[9] Kevork N. Abazajian and Manoj Kaplinghat, "Erratum: Detection of a gamma-ray source in the galactic center consistent with extended emission from dark matter annihilation and concentrated astrophysical emission [phys. rev. d 86, 083511 (2012)]," Phys. Rev. D 87, 129902 (2013).

[10] Chris Gordon and Oscar Macias, "Dark Matter and Pulsar Model Constraints from Galactic Center Fermi-LAT Gamma Ray Observations," (2013), arXiv:1306.5725 [astro-ph.HE]

[11] Vincenzo Vitale and Aldo Morselli (Fermi/LAT Collaboration), "Indirect Search for Dark Matter from the center of the Milky Way with the Fermi-Large Area Telescope," (2009), arXiv:0912.3828 [astro-ph.HE]

[12] V. Vitale, A. Morselli, and Fermi/LAT Collaboration, "Search for Dark Matter with Fermi Large Area Telescope: The Galactic Center," Nuclear Instruments and Methods in Physics Research A 630, 147-150 (2011).

[13] Fabio Iocco, Miguel Pato, Gianfranco Bertone, and Philippe Jetzer, "Dark Matter distribution in the Milky Way: microlensing and dynamical constraints," JCAP 1111, 029 (2011), arXiv:1107.5810 [astro-ph.GA]

[14] Kevork N. Abazajian, "The Consistency of Fermi-LAT Observations of the Galactic Center with a Millisecond
Pulsar Population in the Central Stellar Cluster," JCAP 1103, 010 (2011), arXiv:1011.4275 [astro-ph.HE]

[15] R. S. Wharton, S. Chatterjee, J. M. Cordes, J. S. Deneva, and T. J. W. Lazio, "Multiwavelength Constraints on Pulsar Populations in the Galactic Center," Astrophys. J. 753, 108 (2012), arXiv:1111.4216 [astro-ph.HE].

[16] Chris Gordon and Oscar Macias, "Erratum: Dark Matter and Pulsar Model Constraints from Galactic Center Fermi-LAT Gamma Ray Observations," Phys. Rev. D (2014), arXiv:1306.5725 [astro-ph.HE]

[17] N. Mirabal, "Dark matter versus pulsars: catching the impostor," MNRAS 436, 2461-2464 (2013), arXiv:1309.3428 [astro-ph.HE].

[18] Dan Hooper and Tracy R. Slatyer, "Two Emission Mechanisms in the Fermi Bubbles: A Possible Signal of Annihilating Dark Matter," (2013), arXiv:1302.6589 [astroph.HE]

[19] Wei-Chih Huang, Alfredo Urbano, and Wei Xue, "Fermi Bubbles under Dark Matter Scrutiny. Part I: Astrophysical Analysis," (2013), arXiv:1307.6862 [hep-ph]

[20] Dan Hooper, Ilias Cholis, Tim Linden, Jennifer SiegalGaskins, and Tracy Slatyer, "Millisecond pulsars Cannot Account for the Inner Galaxy's GeV Excess," Phys.Rev. D88, 083009 (2013), arXiv:1305.0830 [astro-ph.HE]

[21] Tim Linden, Elizabeth Lovegrove, and Stefano Profumo, "The Morphology of Hadronic Emission Models for the Gamma-Ray Source at the Galactic Center," Astrophys.J. 753, 41 (2012), arXiv:1203.3539 [astro-ph.HE],

[22] F. Yusef-Zadeh et al., "Interacting Cosmic Rays with Molecular Clouds: A Bremsstrahlung Origin of Diffuse High Energy Emission from the Inner 2deg by 1deg of the Galactic Center," Astrophys.J. 762, 33 (2013), arXiv:1206.6882 [astro-ph.HE],

[23] C. J. Law, F. Yusef-Zadeh, W. D. Cotton, and R. J. Maddalena, "Green Bank Telescope Multiwavelength Survey of the Galactic Center Region," ApJS 177, 255-274 (2008), arXiv:0801.4294.

[24] "Fermi science tools documentation," http://fermi. gsfc.nasa.gov/ssc/data/analysis/documentation/.

[25] P. L. Nolan et al., "Fermi Large Area Telescope Second Source Catalog," Astrophys.J.Suppl. 199, 31 (2012), arXiv:1108.1435 [astro-ph.HE],

[26] F. Aharonian et al., "Discovery of very-high-energy $\gamma$ rays from the Galactic Centre ridge," Nature (London) 439, 695-698 (2006), arXiv:astro-ph/0603021.

[27] Diffuse and Molecular Clouds Science Working Group Fermi Large Area Telescope Collaboration, "Description and caveats for the LAT team model of diffuse gamma-ray emission, version: gll_iem_v02.fit," http://fermi.gsfc.nasa.gov/ssc/data/access/lat/ ring_for_FSSC_final4.pdf (2009).

[28] M. Ackermann et al., "Fermi-lat observations of the diffuse gamma-ray emission: Implications for cosmic rays and the interstellar medium," The Astrophysical Journal 
750, $3(2012)$

[29] A.W. Strong and I.V. Moskalenko, "Propagation of cosmic-ray nucleons in the galaxy," Astrophys.J. 509, 212-228 (1998), arXiv:astro-ph/9807150 [astro-ph]

[30] Andrey E. Vladimirov, Seth W. Digel, Gudlaugur Johannesson, Peter F. Michelson, Igor V. Moskalenko, et al., "GALPROP WebRun: an internet-based service for calculating galactic cosmic ray propagation and associated photon emissions," Comput.Phys.Commun. 182, 11561161 (2011), arXiv:1008.3642 [astro-ph.HE]

[31] Tesla E. Jeltema and Stefano Profumo, "Fitting the Gamma-Ray Spectrum from Dark Matter with DMFIT: GLAST and the Galactic Center Region," JCAP 0811, 003 (2008), arXiv:0808.2641 [astro-ph]

[32] A.A. Abdo et al. (The Fermi-LAT collaboration), "The Second Fermi Large Area Telescope Catalog of Gamma-ray Pulsars," Astrophys.J.Suppl. 208, 17 (2013), arXiv:1305.4385 [astro-ph.HE]

[33] A. A. Abdo et al., "A population of gamma-ray emitting globular clusters seen with the Fermi Large Area Telescope," A\&A 524, A75 (2010), arXiv:1003.3588 [astroph.GA]

[34] The Fermi-LAT Collaboration, "Dark Matter Constraints from Observations of 25 Milky Way Satellite Galaxies with the Fermi Large Area Telescope," ArXiv e-prints (2013), arXiv:1310.0828 [astro-ph.HE],

[35] M. Ackermann et al., "Constraints on the Galactic Halo Dark Matter from Fermi-LAT Diffuse Measurements," Astrophys. J. 761, 91 (2012), arXiv:1205.6474 [astroph.CO]

[36] S. S. Wilks, "The Large-Sample Distribution of the Likelihood Ratio for Testing Composite Hypotheses," Ann.Math.Stat. 9, 60 (1938)

[37] J.R. Mattox, D.L. Bertsch, J. Chiang, B.L. Dingus, S.W. Digel, et al., "The Likelihood Analysis of EGRET Data," Astrophys.J. 461, 396 (1996).

[38] Yasunobu Uchiyama, Stefan Funk, Hideaki Katagiri, Junichiro Katsuta, Marianne Lemoine-Goumard, et al., "Fermi-LAT Discovery of GeV Gamma-ray Emission from the Vicinity of SNR W44," (2012), arXiv:1203.3234 [astro-ph.HE]

[39] M. Ackermann et al. (Fermi-LAT Collaboration), "Detection of the Characteristic Pion-Decay Signature in Supernova Remnants," Science 339, 807 (2013), arXiv:1302.3307 [astro-ph.HE]

[40] F. James and M. Roos, "Minuit: A System for Function Minimization and Analysis of the Parameter Errors and Correlations," Comput.Phys.Commun. 10, 343-367 (1975)

[41] Eric Jones, Travis Oliphant, Pearu Peterson, et al., "SciPy: Open source scientific tools for Python," (2001).

[42] R. Schlickeiser, Cosmic ray astrophysics / Reinhard Schlickeiser, Astronomy and Astrophysics Library; Physics and Astronomy Online Library. Berlin: Springer. ISBN 3-540-66465-3, 2002, $X V+519$ pp. (2002).

[43] H. Bethe and W. Heitler, "On the Stopping of fast particles and on the creation of positive electrons," Proc.Roy.Soc.Lond. A146, 83-112 (1934).

[44] R. Schlickeiser and K. O. Thielheim, "Non-thermal electron bremsstrahlung in the Galactic disk," MNRAS 182, 103-109 (1978).

[45] J. Beringer et al. (Particle Data Group), "Review of Particle Physics (RPP)," Phys.Rev. D86, 010001 (2012).
[46] George B. Rybicki and Alan P. Lightman, "Radiative Processes in Astrophysics," (1985).

\section{Appendix A: Nonthermal Bremsstrahlung Spectrum}

In this appendix we discuss the relevant synchrotron and bremsstrahlung formula which were used in the analysis of the current article and Ref. 22]. As these formulas are only briefly alluded to in Ref. [22] we provide more details here which are based on notes kindly supplied to us by Prof. Mark Wardle.

Relativistic cosmic ray electrons that are deflected in the Coulomb field of nuclei in molecular clouds at the Galactic Center emit bremsstrahlung $\gamma$-ray photons 42 . In this region the ionized gas component contribution to the radiation process can be neglected [42]. By considering this the differential cross section for the bremsstrahlung interaction [43, 44] can be written as ${ }^{2}$

$$
\begin{aligned}
\sigma\left(E_{\gamma}, \gamma\right)= & \frac{3}{8 \pi E_{\gamma}} \alpha \sigma_{T} \Phi_{\mathrm{H}}\left[\frac{4}{3}-\frac{4}{3} \frac{E_{\gamma}}{E}+\left(\frac{E_{\gamma}}{E}\right)^{2}\right] \\
& \mathrm{cm}^{2} \mathrm{eV}^{-1}
\end{aligned}
$$

where $E_{\gamma}$ is the photon energy, $E=\gamma m c^{2}$ the relativistic electron energy, $\alpha=1 / 137.0$ the fine structure constant, $\sigma_{T}=6.652 \times 10^{-25} \mathrm{~cm}^{2}$ the Thomson cross section and $\Phi_{\mathrm{H}} \simeq 45$ the scattering function assumed to be in the strong-shielding limit which is appropriate for relativistic electrons. We take the invariant electron mass to be $m=$ $9.109 \times 10^{-28} \mathrm{~g}$ [45] and for the speed of light we use $c=2.998 \times 10^{10} \mathrm{~cm} / \mathrm{s}$.

The nonthermal electron bremsstrahlung omnidirectional source function produced by a single relativistic electron in a medium dominated by atomic and molecular hydrogen nuclei of corresponding number density $n_{\mathrm{H}}=n_{\mathrm{HI}}+2 n_{\mathrm{H}_{2}}$ is given by 42

$$
\begin{aligned}
q\left(E_{\gamma}\right)= & c n_{\mathrm{H}} \int_{E_{\mathrm{L}}}^{+\infty} d \gamma n(\gamma) \sigma\left(E_{\gamma}, \gamma\right) \\
& \text { photons } \mathrm{cm}^{-3} \mathrm{~s}^{-1} \mathrm{eV}^{-1}
\end{aligned}
$$

where $E_{\mathrm{L}}=\max \left(E_{\gamma}, E_{1}\right)$ and $E_{1}=\gamma_{1} m c^{2}$ represents a low-energy cutoff in the electron distribution. The energy distribution function $n(\gamma)$ of the radiating relativistic electrons is assumed to follow a broken power law of the form

$$
n(\gamma)=\left\{\begin{array}{ll}
n_{1} \gamma^{-p_{1}} & \text { if } 1 \leq \gamma \leq \gamma_{b} \\
n_{2} \gamma^{-p_{2}} & \text { if } \gamma \geq \gamma_{b},
\end{array} \quad \mathrm{~cm}^{-3}\right.
$$

with $n_{1} \gamma_{b}^{-p_{1}}=n_{2} \gamma_{b}^{-p_{2}} \equiv n_{b}$ and $E_{b}=\gamma_{b} m c^{2}$ the break energy. After substituting Eq. A1 and A3 into

\footnotetext{
${ }^{2}$ We note that Eq. 4.4.1 of Ref. 42 is missing a factor of $1 / E_{\gamma}$.
} 
Eq. A2 and solving the corresponding integrals we get for the omnidirectional source function

$$
\begin{gathered}
q\left(E_{\gamma}\right)=\frac{3 \alpha \sigma_{T}}{8 \pi} \Phi_{\mathrm{H}} n_{\mathrm{H}} \frac{n_{b}}{m c} J\left(E_{\gamma} / E_{b}\right) \\
\text { photons } \mathrm{cm}^{-3} \mathrm{~s}^{-1} \mathrm{eV}^{-1},
\end{gathered}
$$

where

$$
J(x)= \begin{cases}{\left[I_{p_{1}}(1)-I_{p_{1}}(1 / x)\right] x^{-p_{1}}+I_{p_{2}}(1 / x) x^{-p_{2}}} & \text { for } x<1 \\ I_{p_{2}}(1) x^{-p_{2}} & \text { for } x \geq 1\end{cases}
$$

and

$$
\begin{aligned}
I_{p_{i}}(x)= & \frac{1}{3} x^{-p_{i}}\left(\frac{3}{x+p_{i} x}+\frac{4 x}{p_{i}-1}-\frac{4}{p_{i}}\right) \\
& \text { for } i=1,2 .
\end{aligned}
$$

In Ref. 22 it was argued that the morphological distribution of diffuse radiation from the GC measured at 1.45 $\mathrm{GHz}, \mathrm{GeV}$ and $\mathrm{TeV}$ energies is correlated. Importantly, in that work it was assumed that all the synchrotron emitting electrons are interacting with the molecular clouds. It is thus interesting to find an expression for the bremsstrahlung spectrum in terms of the synchrotron flux.

Synchrotron emission at frequency $\nu$ (taken to be the characteristic synchrotron radiation frequency) is associated with particles of energy (see Eq. 4.1.9 of [42])

$$
\begin{aligned}
E_{\nu} & =\gamma_{\nu} m c^{2} \\
& =\left(\frac{4 \pi m c \nu}{3 e B}\right)^{1 / 2} m c^{2} \\
& =7.89\left(\frac{B}{\mu \mathrm{G}}\right)^{-1 / 2}\left(\frac{\nu}{\mathrm{GHz}}\right)^{1 / 2} \mathrm{GeV} .
\end{aligned}
$$

where the electron charge is $e=4.803 \times 10^{-10}$ statcoulomb. Eq. A7 can be rewritten as

$$
\gamma_{\nu}=\left(\frac{\nu}{\nu_{B}}\right)^{1 / 2} \text { where } \nu_{B}=\frac{3 e B}{4 \pi m c}=4.20\left(\frac{B}{\mu \mathrm{G}}\right)
$$

We therefore obtain that particles radiating at the synchrotron break frequency $\nu_{b}$ obey the relation

$$
\nu_{b}=\nu_{B} \gamma_{b}^{2} \quad \mathrm{~Hz}
$$

The synchrotron emission coefficient resulting from an electron spectrum that is a simple power law can be obtained from Eq.(6.36) of Ref. 46] which can be rewritten as

$j_{\nu}=\frac{1}{\sqrt{3}} f_{j}(p) \frac{e^{2}}{c} \nu_{B} \gamma_{\nu} n\left(\gamma_{\nu}\right) \quad \operatorname{erg~cm}^{-3} \operatorname{ster}^{-1} \mathrm{~s}^{-1} \mathrm{~Hz}^{-1}$

where

$$
f_{j}(p)=\frac{2^{(p-1) / 2}}{p+1} \Gamma\left(\frac{3 p-1}{12}\right) \Gamma\left(\frac{3 p+19}{12}\right),
$$

and $\Gamma(z)$ is the Gamma function. Using Eq. A10 we can estimate the emission coefficient for a broken power law at the synchrotron break frequency

$$
j_{b}=\tilde{j} n_{b} \quad \operatorname{erg} \mathrm{cm}^{-3} \operatorname{ster}^{-1} \mathrm{~s}^{-1} \mathrm{~Hz}^{-1}
$$

where

$$
\tilde{j}=\frac{1}{\sqrt{3}} \bar{f}_{j}(p) \frac{e^{2}}{c} \sqrt{\nu_{B} \nu_{b}} \quad \text { erg } \operatorname{ster}^{-1} \mathrm{~s}^{-1} \mathrm{~Hz}^{-1},(\mathrm{~A}
$$

and

$$
\bar{f}_{j}(p)=\frac{1}{2}\left[f_{j}\left(p_{1}\right)+f_{j}\left(p_{2}\right)\right] .
$$

where $p_{1}$ and $p_{2}$ are the broken power law spectral indices before and after the break.

Then the spectral value of synchrotron radiation from a source of volume $V$ at a distance $d$ at the break frequency is given by

$$
S_{b}=4 \pi \frac{\tilde{j} n_{b} V}{4 \pi d^{2}} \quad \mathrm{Jy} .
$$

Finally, the source function given in Eq. A4 is multiplied by a factor $V$ to get the photon luminosity spectrum and then divided by $4 \pi d^{2}$ to obtain the bremsstrahlung photon flux spectrum and using the results of Eq A15 we thus get for the bremsstrahlung $\gamma$-ray spectrum

$$
\begin{gathered}
\frac{d N_{\text {brem }}}{d E_{\gamma}}=\frac{3 \alpha \sigma_{T}}{32 \pi^{2} \tilde{j}} \Phi_{\mathrm{H}} n_{\mathrm{H}} \frac{S_{b}}{m c} J\left(E_{\gamma} / E_{b}\right) \\
\text { photons } \mathrm{erg}^{-1} \mathrm{~cm}^{-2} \mathrm{~s}^{-1},
\end{gathered}
$$

where $J\left(E_{\gamma} / E_{b}\right)$ is given by Eq. A5 
Appendix B: Gamma-Ray Excess Data

\begin{tabular}{|c|c|c|c|c|c|}
\hline$E_{\min }[\mathrm{GeV}]$ & $E_{\max }[\mathrm{GeV}]$ & $d N / d E\left[\mathrm{GeV}^{-1} \mathrm{~cm}^{-2} \mathrm{~s}^{-1}\right]$ & Stat. Error $\left[\mathrm{GeV}^{-1} \mathrm{~cm}^{-2} \mathrm{~s}^{-1}\right]$ & Syst. Error $\left[\mathrm{GeV}^{-1} \mathrm{~cm}^{-2} \mathrm{~s}^{-1}\right]$ & TS \\
\hline 0.30 & 0.40 & $1.11 \times 10^{-6}$ & $6.41 \times 10^{-7}$ & $3.92 \times 10^{-7}$ & 3.06 \\
\hline 0.40 & 0.54 & $7.17 \times 10^{-7}$ & $6.71 \times 10^{-8}$ & $2.84 \times 10^{-7}$ & 5.93 \\
\hline 0.54 & 0.72 & $4.28 \times 10^{-7}$ & $4.54 \times 10^{-8}$ & $1.26 \times 10^{-7}$ & 10.45 \\
\hline 0.72 & 0.97 & $3.02 \times 10^{-7}$ & $3.05 \times 10^{-8}$ & $7.98 \times 10^{-8}$ & 25.21 \\
\hline 0.97 & 1.29 & $1.95 \times 10^{-7}$ & $2.57 \times 10^{-8}$ & $3.80 \times 10^{-8}$ & 49.1 \\
\hline 1.29 & 1.73 & $1.23 \times 10^{-7}$ & $1.81 \times 10^{-8}$ & $1.83 \times 10^{-8}$ & 73.51 \\
\hline 1.73 & 2.32 & $5.44 \times 10^{-8}$ & $1.48 \times 10^{-8}$ & $9.38 \times 10^{-9}$ & 63.94 \\
\hline 2.32 & 3.11 & $3.39 \times 10^{-8}$ & $7.25 \times 10^{-9}$ & $4.35 \times 10^{-9}$ & 84.03 \\
\hline 3.11 & 4.16 & $1.33 \times 10^{-8}$ & $6.11 \times 10^{-9}$ & $1.98 \times 10^{-9}$ & 43.3 \\
\hline 4.16 & 5.57 & $3.42 \times 10^{-9}$ & $3.17 \times 10^{-9}$ & $3.17 \times 10^{-9}$ & 10.46 \\
\hline 5.57 & 7.47 & $2.26 \times 10^{-9}$ & $6.97 \times 10^{-10}$ & $3.01 \times 10^{-10}$ & 14.14 \\
\hline 7.47 & 10.00 & $1.21 \times 10^{-9}$ & $2.51 \times 10^{-10}$ & $2.40 \times 10^{-10}$ & 12.82 \\
\hline 10.00 & 100.00 & - & - & - & 0.12 \\
\hline
\end{tabular}

TABLE V. NFW N.2 $^{2}$ spectrum and corresponding statistical and systematic errors using model 1 in Table I The spectral points $d N / d E$ were obtained at the logarithmic midpoint of each band.

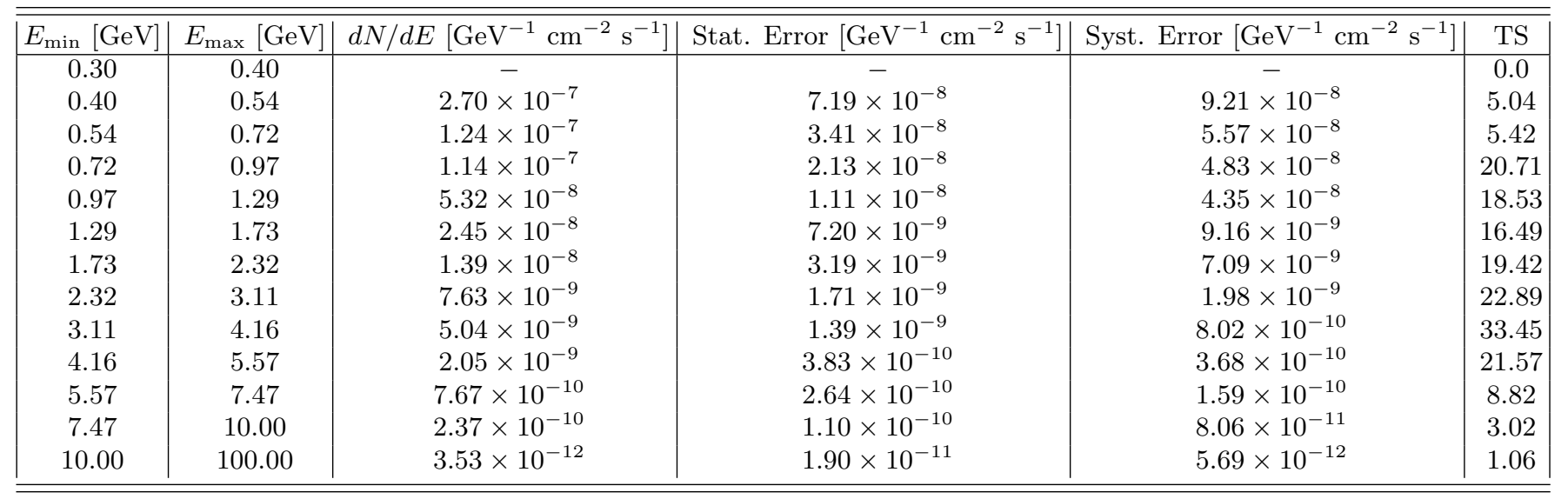

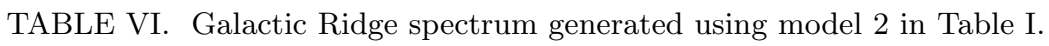

